\title{
A randomized clinical trial of metacognitive therapy and nortriptyline for anxiety, depression, and difficulties in emotion regulation of patients with functional dyspepsia
}

\author{
Sepideh Batebi, ${ }^{1}$ Abbas Masjedi Arani, ${ }^{1}$ Mahdi Jafari, ${ }^{1}$ Amir Sadeghi, ${ }^{2}$ Mohsen Saberi Isfeedvajani, ${ }^{3}$ \\ Mohammad Hasan Davazdah Emami ${ }^{4}$
}

${ }^{1}$ Department of Clinical Psychology, Faculty of Medicine, Shahid Beheshti University of Medical Sciences; ${ }^{2}$ Gastroenterology and Liver Diseases Research Center, Research Institute for Gastroenterology and Liver Diseases. Shahid Beheshti University of Medical Sciences; ${ }^{3}$ Medicine, Quran and Hadith Research Center \& Department of Community Medicine, Faculty of Medicine, Baqiyatallah University of Medical Sciences, Tehran; ${ }^{4}$ Department of Health Psychology, Isfahan University of Medical Science, Isfahan, Iran

\begin{abstract}
Functional Dyspepsia (FD) as a psychosomatic disorder is an upper gastrointestinal tract disease without organic pathogenesis causes. The psychopathological nature of this disease and its high correlation with anxiety and depression implies the need for psychological interventions. The purpose of the present study is to compare the efficacy of Metacognitive Therapy (MCT) and medication for the symptoms of anxiety, depression, and difficulties in emotion regulation in patients with FD. In a randomized clinical trial, 65 patients with FD were recruited during their visit to gastroenterology clinics. These patients were randomly assigned to three groups to receive MCT, nortriptyline treatment, and controls. They were treated for 10 weeks and followed up three months later. The instruments used in this study were Hamilton anxiety and Depression Rating Scale (HAM-A

Correspondence: Abbas Masjedi Arani, Department of Clinical Psychology, Faculty of Medicine, Shahid Beheshti University of Medical Sciences, Tehran, Iran.

Tel.: +9823031548 .

Fax: +9823031548 .

E-mail: dr.abbas.masjediarani98@gmail.com

Acknowledgments: We would like to express our sincere thanks and appreciation to all respected staff and doctors of Research Institute for Gastroenterology and Liver Diseases of Shahid Beheshti University and gastroenterology clinics related to Shahid Beheshti Uniand HDRS) and difficulties in emotion regulation scale. The results were analyzed using repeated measure analysis by SPSS (19IBM). Data analysis showed statistically significant differences in the variables of depression, anxiety among MCT, nortriptyline treatment, and controls at pre-test, post-test and follow-up phases. Moreover, MCT had a better and more persistent effect on anxiety compared to nortriptyline treatment, as well as a better efficacy in treating anxiety and depression symptoms compared to the controls. MCT demonstrated better efficacy in treating anxiety symptoms compared to nortriptyline treatment and controls.
\end{abstract} versity of Medical Sciences, Dr. M. R. Zali, Dr. M. Iranshahi, Dr. S. Abdi, Dr. H. Malekpour for their time and invaluable support.

Citation: Batebi, S., Masjedi Arani, A., Jafari, M., Sadeghi, A., Saberi Isfeedvajani, M., \& Davazdah Emami, M.H. (2020). A randomized clinical trial of metacognitive therapy and nortriptyline for anxiety, depression, and difficulties in emotion regulation of patients with functional dyspepsia. Research in Psychotherapy: Psychopathology, Process and Outcome, 23(2), 157-166. doi: 10.4081/ripppo.2020.448

Conflict of interest: the authors declare no potential conflict of interest.

Ethical approval and consent to participate: the code of ethics IR.SBMU.MSP.REC.1396.756. Furthermore, the study was registered (2019) with the IID: IRCT20190312043036N1.

Received for publication: 31 December 2019

Accepted for publication: 21 August 2020.

This work is licensed under a Creative Commons Attribution NonCommercial 4.0 License (CC BY-NC 4.0).

${ }^{\circ}$ Copyright: the Author(s), 2020

Licensee PAGEPress, Italy

Research in Psychotherapy:

Psychopathology, Process and Outcome 2020; 23:157-166

doi:10.4081/ripppo.2020.448
Key words: dyspepsia; psychotherapy; anxiety; depression; emotion.

\section{Introduction}

FD is a heterogeneous condition that may Include Postprandial Distress Syndrome (PDS) and meal-related symptoms, such as saturation, early satiety, bloating and Epigastric Pain Syndrome (EPS); however, EPS is characterized by some non-meal-related symptoms including epigastric pain and heartburn (Schmulson \& Drossman, 2017).

The disease is defined as a multifactorial disorder, and several hypotheses have been proposed to determine the etiology of this disorder; one of them is the psychological hypothesis. In this hypothesis, mental disorders, such as depression, anxiety or psychosomatic disorders are considered as the key factors of FD. Additionally, in many cases in the literature, FD has been considered as a psychosomatic disorder (Tanum, \& Malt, 2001; Talley, Ruff, Jiang, \& Jung, 2008; Chen, Luo, \& Chang, 2010; Mahadeva, \& Goh, 2011; Filipović et al., 2013). 
Complex interactions and emotional variables contribute to the emergence of FD symptoms. Particularly, emotional stress is a key factor in the pathology of FD and is considered as a pathogenesis factor of FD. Exposure to stress triggers the entire activation of the hypothalamicpituitary-adrenal axis and Autonomic Nervous System (ANS), which is associated with the release of Corticotrophin Hormone (CRH), and this leads to psychosomatic symptoms related to the gastrointestinal system (Jones, Dilley, Drossman, \& Crowell, 2006; Taché, \& Bonaz, 2007; Sahan et al., 2018).

According to the studies that have been conducted on $\mathrm{FD}$, there is a higher prevalence of anxiety and depression symptoms, comorbidity of neurotic personality traits and frequent somatic complaints (Filipović et al., 2013; Tanum, \& Malt, 2001; Mahadeva, \& Goh, 2011).

The results of some recent studies have indicated that the emotion regulation impairment in patients with Generalized Anxiety Disorder (GAD) affects the formation and persistence of this disorder (Mennin, Heimberg, Turk, \& Fresco, 2002; Mennin, Turk, \& Heimberg, 2004; Mennin, Heimberg, Turk, \& Fresco, 2005; Mennin, Holaway, Fresco, Moore, \& Heimberg, 2007).

Patients with GAD have some deficiency in monitoring, perception, and regulation of emotions especially involving in goal-directed behaviors (Salters-Pedneault, Tull, \& Roemer, 2004; Turk, Heimberg, \& Luterek, 2005; Salters-Pedneault, Roemer, Tull, Rucker, \& Mennin, 2006). Accordingly, because of the higher rates of anxiety disorder in FD patients (Li, Nie, Sha, \& Su, 2002; Aro et al., 2009; Van Oudenhove et al., 2007; Wu, 2012), it is expected that FD patients are also affected by emotion regulation impairment.

Emotion regulation is defined as the process of initiating, maintaining, modulating or changing the incidence, severity or persistence of internal feelings and emotions that are related to social, psychological and physiological processes, for accomplishing one's goals (Gross, 2001; Gross, \& Thompson, 2007).

Very few studies have been conducted on the effects of psychotherapy on improving the symptoms of anxiety and depression of patients with FD. Drossman et al. (2003) suggested that cognitive behavioral therapy and stress management using relaxation techniques can effectively reduce the symptoms of anxiety, depression, and pain in patients with gastrointestinal diseases. It was shown that flexible coping psychotherapy can improve the symptoms of dyspepsia and anxiety (Cheng, Yang, Jun, \& Hutton, 2007).

Moreover, it was shown that intensive medical treatment with Cognitive-Behavioral Therapy (CBT) can reduce the symptoms of anxiety and depression (Haag et al, 2007). Other types of psychotherapy that have studied only the effects of interventions on the somatic symptoms of FD include hypnotherapy (Calvert, Houghton, Cooper, Morris, \& Whorwell, 2002), psychodynamic interpersonal psychotherapy (Hamilton et al., 2000), cognitive therapy (Haug, Wilhelmsen, Svebak, Berstad, \& Ursin, 1994; Haug, 2002), short-term psychodynamic psychotherapies (STPP) (Faramarzi et al., 2013), group psychotherapy (Orive et al., 2015), stress management with a cognitivebehavioral approach (Dehghanizade, Zargar, Honarmand, Kadkhodaie, \& Baygi, 2015), and stress management training (Bagherian, Pourkazem, Nouri, \& Adibi, 2009).

MCT particularly addresses worry in patients with anxiety disorder. Metacognition deals with psychological structures; knowledge, events and informational processes that are associated with the control, modification and interpretation of thought (Wells, 2002; Wells, 2011). Based on this theory, positive and negative beliefs about worry increase worry and anxiety due to interfering with self-regulation processes. Moreover, coping behaviors including avoiding intrusive thoughts, distraction and suppression of anxietyprovoking thoughts, all reinforce positive and negative metacognitive beliefs, and thereby increase worry (Davis, \& Valentiner, 2000; Wells, 2002; Wells, 2011).

Accordingly, the purpose of the present study is to compare MCT with nortriptyline treatment and controls in symptoms of anxiety, depression, and difficulties in emotion regulation in patients with FD.

\section{Materials and Methods}

In the first place, 65 patients with FD were recruited during their visit to the research center of Gastroenterology and Liver Diseases of Taleghani Hospital affiliated to Shahid Beheshti University of Medical Sciences, Tehran. These patients were then interviewed by gastroenterologists based on Rome-IV diagnostic criteria for gastrointestinal disorders. Moreover, these patients underwent endoscopy and H-Pylori tests for rejecting underlying organic causes of dyspepsia.

Patients' inclusion criteria were: i) having no physical cause for dyspepsia; ii) no other gastrointestinal comorbidities; iii) no severe psychiatric disorders; iv) not taking other medications; v) no drug dependency; vi) no attendance in other psychotherapy sessions and practices such as yoga and meditation; vii) age between 18 and 55 years; viii) reading and writing ability. Patients' exclusion criteria were: i) non-compliance with treatment protocols; ii) noncompliance with medical treatment as usual for all patients; iii) initiation to take medications out of treatment protocols; iv) initiation of drug use; v) absence in two consecutive or three intermittent psychotherapy sessions; vi) unwillingness to participate in the therapy for any reason during the study.

After signing written consent, all participated patients were consulted in a session on healthy diet, regular exercise and taking omeprazole (omeprazole, $20 \mathrm{mg}$, once daily) from Sobhan Darou Co. (Tehran, Iran) and domperidone (motidon, $10 \mathrm{mg}$, once daily) from Abidi pharmacy Co. (Tehran, Iran) as the standard medical treatment. Then, in a simple random sampling process, 
the twenty patients were assigned to MCT and nortriptyline groups while the control group contained 25 patients because of the higher probability of patient dropout in this group for the reason of no specific treatment more than medical treatment as usual. MCT for generalized anxiety disorder based on Well's treatment protocol (Wells, 2011) in 10 (45-minute) sessions over 10 weeks. The brief contents of the sessions were as follows: Session 1: case formulation, acquainting with the metacognitive model, beginning to challenge metacognitive beliefs and the first detached mindfulness exercise, Session 2: continuation of challenging uncontrollability negative metacognitive beliefs and employing cognitive techniques, Session 3: continuation of challenging uncontrollability beliefs, employing behavioral techniques to remove avoidant behaviors, Session 4: challenging danger beliefs, Session 5: continuation of challenging metacognitive beliefs about danger with the help of behavioral techniques, Session 6: reversing residual maladaptive strategies, Session 7: initiating to challenge positive metacognitive beliefs using behavioral techniques, Session 8: continuing to challenge positive metacognitive beliefs, Session 9: continuation of treating residual symptoms and forming a new processing style, Session 10: relapse prevention and initiate to work on the new plan. For the other intervention group prescribed nortriptyline (nortriptyline, $25 \mathrm{mg}$, once daily, over 10 weeks) from Sobhan Darou Co. (Tehran, Iran) and for controls only took omeprazole and domperidone as the standard medical treatment.

The first phase of evaluation involved the examination of patients before initiating the treatment by interviewing them based on Rome-IV diagnostic criteria for functional gastrointestinal disorders by a gastroenterologist. Dyspeptic patients underwent endoscopy and H-Pylori tests to exclude the underlying organic dyspepsia. In line with the gastroenterology interview, we assessed the patients by Leeds Dyspepsia Questionnaire (LDQ) to increase the incremental reliability and validity of the interview.

Next, structured psychiatric clinical interviews were conducted by a clinical psychologist using the diagnostic and statistical manual of mental disorders, fifth edition (DSM 5) to evaluate patients and exclude those with severe mental and personality disorders. Then, the clinical assessment was carried out to determine the levels of depression and anxiety of the patients based on Hamilton anxiety and depression scale. The patients were given a questionnaire on difficulties in emotion regulation to assess their emotion regulation dysfunction. Moreover, partial treatment compliance with medical treatment as usual assessed by interview during the sessions.

The assessment of anxiety, depression, and difficulties in emotion regulation was conducted at pre-, post-treatment phase and three months after treatment. In the process of the study, three patients from the MCT group dropped out due to some family problems and failure to attend the last treatment sessions. Four patients from the nortriptyline treatment group dropped out because of unwillingness to take medications due to the side effects and lack of satisfactory results. Moreover, 10 patients from the control group dropped out due to the low efficacy of standard medical treatment in improving symptoms such that meet their expectation.

It is worth mentioning that the present study has been derived from a dissertation with the code of ethics IR.SBMU.MSP.REC.1396.756, which was received, prior to the study (2018), from the Research Committee of Shahid Beheshti University of Medical Sciences, Tehran, Iran. Furthermore, the study was registered (2019) in Iranian Registry of Clinical Trials with the ID: IRCT20190312043036N1 which is available on the website: http://www.irct.ir.

\section{Measurements}

Clinical interview according to Rome-IV diagnostic criteria for functional gastrointestinal disorders:

These criteria measure some clinical symptoms of FD including gastrointestinal and reflux symptoms. This was conducted by gastroenterologists at pre-treatment, posttreatment and three months after treatment.

\section{Endoscopy}

To distinguish between FD and other types of dyspeptic disorders with underlying organic causes, patients underwent upper gastrointestinal endoscopy at the beginning of the study and after the interview based on Rome-IV diagnostic criteria.

Structured clinical interview for Diagnostic and statistical manual of mental disorders (DSM-5) clinician version (SCID-5/CV) (American Psychiatric Association, 2013; First, 2014): This interview was conducted at the pre-treatment phase to evaluate patients and exclude those with severe mental and personality disorders based on DSM 5.

\section{Leeds Dyspepsia Questionnaire (LDQ)}

The questionnaire has eight items that measure Functional Dyspepsia (FD) symptoms according to their frequency and severity. The items related to the symptoms in the upper gastrointestinal tract include epigastric pain, retrosternal pain, dysphagia, regurgitation, belching, nausea, vomiting and bloating. The LDQ has a range of scores from 0 to 40; lower scores indicate fewer symptoms, while higher scores indicate more severe dyspepsia. The internal consistency of the questionnaire in a population of 166 Asian patients was 0.80 , test-retest correlation 0.98 and validity 0.77 , which indicates desirable reliability and validity (Mahadeva, Chan, Mohazmi, Sujarita, \& Goh, 2011). Another study reported a sensitivity of $80 \%$ and a specificity of $79 \%$ in the general population (Moayyedi et al., 1998). In Iranian sample, the internal consistency of the questionnaire ranged from 0.80 to 0.89 (for a sample of 93 with FD and a sample of 67 partici- 
pants without dyspepsia) and test-retest reliability was 0.96 , which indicate good consistency and validity of the questionnaire (Batebi et al., 2019).

\section{Hamilton anxiety rating scale (HAM-A; Hamilton, 1959)}

Hamilton anxiety rating scale (HAM-A) is a clinicianrated scale that included 14 items and each item was associated with specific anxiety symptoms. The reliability and concurrent validity of this scale were reported to be sufficient (Maier, Buller, Philipp, \& Heuser, 1988). This scale is considered as an original questionnaire in evaluating anxiety with great validity. The test-retest reliability of this questionnaire was reported to be 0.81 in an Iranian sample (Salmani, Hasani, Mohammad-Khani, \& Karami, 2014).

\section{Hamilton Depression Rating Scale (HDRS; Hamilton, 1960)}

The therapist used Hamilton Depression Rating Scale (HDRS) (21-item) to evaluate the severity of depression. The validity and reliability of this scale were reported to range from 0.91 to 0.94 using Cronbach's alpha. The credibility of this scale was also reported to range from 0.65 to 0.90 (Todorova, \& Velikova, 2012). Accordingly, HRSD is considered as one of the main scales in the study of depression. The sensitivity and specificity of this test were 0.93 and 0.98, respectively (Cusin, Yang, Yeung, \& Fava, 2009). Test-retest reliability coefficients in an Iranian sample with HAMA were reported as $85 \%$ and $89 \%$ (Mokhber, AzarPajooh, \& Asghari, 2013), and the inter-rater reliability as 0.95 (Ebrahimi, Kheyr, Nasiri, \& Barnamanesh, 2017).

\section{Difficulties in emotional regulation scale}

The Difficulties in Emotional Regulation Scale (DERS) developed by Gratz and Roemer (2004) is a 36item scale with a total score and six subscale scores associated with six different dimensions of difficulties in emotion regulation. The reliability and validity of the total score of this scale had a Cronbach's alpha coefficient of 0.93 , and that of all subscales had alpha coefficients greater than 0.80 . Furthermore, the test-retest reliability of the scale over a 4-8-week period was reported to be acceptable in the Iranian sample, the internal consistency of the total scale was obtained 0.92 using Cronbach's alpha method (Molazadeh, \& Yavari, 2016).

\section{Data analysis}

According to the research design, a randomized clinical trial was considered with a control group, and the trial included pre-test, post-test and follow-up. Descriptive mean and standard deviation were used to describe the data, and the repeated measures analysis was used for data analysis in SPSS (19-IBM Corp., Armonk, N.Y., USA) software.

\section{Results}

Table 1 shows the comparison of mean, standard deviation and frequency distribution of demographic characteristics (age, sex, education, marital status and occupational status) among the groups of MCT $(17,35 \%)$, nortriptyline treatment $(16,33 \%)$ and control $(15,31 \%)$. In general, patients were between 20 and 50 years of age $(\mathrm{MD}=37.46$, $\mathrm{SD}=8.87)$, predominantly female $(41,85.4 \%)$, married $(34,70.8 \%)$, high school graduates/dropouts $(25,52.1 \%)$ and mostly unemployed (26, 54.2\%). Frequency distribution of age was normal and the distributions of other demographic variables were non-normal. A Kruskal-Wallis test was used for non-normal variables to assess the statistical significance of differences between the three groups. The results of the assessment of the mean differences among the demographic variables of the groups are shown in Table 1. These results show that there is no statistically significant difference among the means of the demographic variables in the three groups, and these groups are homogeneous in terms of demographic characteristics.

Kolmogorov-Smirnov normality test for the scores of anxiety, depression and emotional processing showed no statistical significance, so all the studied variables

Table 1. Mean, standard deviation and frequency of demographic variables.

\begin{tabular}{|c|c|c|c|}
\hline Demographic variable & MCT ${ }^{\S}$ & Nortriptyline & Control \\
\hline Mean age (SD) & $34.88(8.9)$ & $38.31(8.9)$ & $39.47(8.6)$ \\
\hline Female & $13(76.5 \%)$ & $14(87.5 \%)$ & $14(93.3 \%)$ \\
\hline Male & $4(23.5 \%)$ & $2(12.5 \%)$ & $1(6.7 \%)$ \\
\hline High school graduates/dropouts & $10(58.8 \%)$ & $8(50 \%)$ & $7(46.7 \%)$ \\
\hline Upper high school graduates & $7(41.2 \%)$ & $8(50 \%)$ & $8(53.3 \%)$ \\
\hline Single & $7(41.2 \%)$ & $4(25 \%)$ & $3(20 \%)$ \\
\hline Married & $10(58.8 \%)$ & $12(75 \%)$ & $12(80 \%)$ \\
\hline Unemployed & $9(52.9 \%)$ & $7(43.8 \%)$ & $10(66.7 \%)$ \\
\hline Employed & $8(47.1 \%)$ & $9(56.3 \%)$ & $5(33.3 \%)$ \\
\hline
\end{tabular}


were normal $(\mathrm{P}<0.05)$ and a parametric test such as repeated measures analysis of variance can be used for data analysis.

The mean and standard deviation of the three groups (metacognitive therapy, nortriptyline-treated and control group) for the scores of Hamilton anxiety and depression scales and the total score of difficulties in emotion regulation at pre-test, post-test and follow-up phases are shown in Table 2. According to this table, for the mentioned variables, the patients in the metacognitive therapy and control group had, respectively, the lowest and highest means at post-treatment and follow-up phases.

Due to the statistical significance of the Mauchly test in all three variables of Hamilton anxiety and depression and difficulties in emotion regulation, which indicated heterogeneity of variance and covariance matrices, the hypothesis of sphericity was rejected. Therefore, the results of the Greenhouse-Geisser test were investigated for these variables. Table 3 shows the results of GreenhouseGeisser correction for within-subjects effects. The results in this table show the statistical significance of the scores of anxiety, depression and difficulties in emotion regulation $(\mathrm{P}<0.05)$ at different phases, i.e., pre-test, post-test and follow-up. This implies that the mean scores of variables have changed over time.
Moreover, the between-subjects effects showed that the effects of groups on anxiety $(\mathrm{F}=1007.21)$, depression $(\mathrm{F}=1250.71)$ and the total score of difficulties in emotion regulation $(\mathrm{F}=1448.69)$ were statistically significant $(\mathrm{P}<0.05)$. This implied that the treatments were effective on the scores of the variables at least in two groups, at post-test and follow-up phases. In addition, the eta effect size of the groups obtained for the scores of Hamilton anxiety and depression and difficulties in emotion regulation were, respectively, $0.56,0.61$ and 0.11 , and the values of the time-group interaction effect size were 0.95 , 0.96 and $0.97(\mathrm{P}<0.05)$. These results imply that the three scores were highly affected over time.

The results in Table 4 show the pairwise comparisons of the groups. As can be seen, there was a significant difference in Hamilton anxiety scale scores between the MCT and nortriptyline, as well as the MCT and control group. Moreover, in the Hamilton depression scale scores, there was a significant difference between $\mathrm{MCT}$ and controls. However, there was no significant difference between the scores of this variable in the MCT and nortriptyline group. Additionally, no significant difference was found in emotion regulation difficulties scores in between-group comparisons $(\mathrm{P}<0.05)$.

Table 5 presents the pairwise comparisons of the

Table 2. Mean and standard deviation of three groups for the scores of anxiety, depression and the total score of difficulties in emotion regulation at pre-test, post-test and follow-up phases.

\begin{tabular}{lcccc}
\hline Variables & Phases & $\begin{array}{c}\text { MCT } \\
\text { Mean (SD) }\end{array}$ & $\begin{array}{c}\text { Nortriptyline } \\
\text { Mean (SD) }\end{array}$ & $\begin{array}{c}\text { Control } \\
\text { Mean (SD) }\end{array}$ \\
\hline HAMA $^{\mp}$ score & Pretest & $32.12(8.90)$ & $34.69(6.61)$ & $35.60(6.87)$ \\
& Post-test & $14.12(6.07)$ & $23.63(7.57)$ & $36.13(6.52)$ \\
& Follow up & $13.29(5.75)$ & $24.50(7.00)$ & $36.60(6.70)$ \\
\hline HADS $^{\ddagger}$ score & Pretest & $26.53(6.53)$ & $24.69(5.19)$ & $31.13(5.30)$ \\
& Post-test & $14.29(3.72)$ & $19.13(5.56)$ & $32.20(5.87)$ \\
& Follow up & $13.65(3.27)$ & $20.50(5.42)$ & $31.73(5.49)$ \\
\hline DERS $^{\ddagger}$ score & Pretest & $132.06(18.00)$ & $115.63(24.35)$ & $105.67(14.71)$ \\
& Post-test & $79.41(25.75)$ & $106.13(24.66)$ & $107.20(17.31)$ \\
& Follow up & $73.00(23.72)$ & $108.69(23.99)$ & $105.40(14.58)$ \\
\hline
\end{tabular}

$\S$, Metacognitive therapy; $\uparrow$, Hamilton anxiety scale; $¥$, Hamilton depression scale; £, Difficulties in emotion regulation scale.

Table 3. Repeated measures analysis of within-group effects on the scores of Hamilton anxiety and depression and difficulties in emotion regulation.

\begin{tabular}{lccccccc}
\hline Variables & Source & Sum of square & Df & Mean square & F & P-value & Partial Eta squared \\
\hline HAMA $^{*}$ score & Time & 2834.89 & 1.1 & 2411.39 & 82.34 & 0.000 & 0.64 \\
& Time*group & 1977.30 & 2.3 & 840.96 & 28.71 & 0.000 & 0.56 \\
& Error & 1549.17 & 52.9 & 29.28 & - & - & - \\
\hline HADS $^{*}$ score & Time & 977.45 & 1.2 & 784.78 & 57.24 & 0.000 & 0.56 \\
& Time*group & 987.00 & 2.4 & 396.22 & 28.90 & 0.000 & 0.56 \\
& Error & 768.36 & 56.04 & 13.70 & - & - & - \\
\hline DERS $^{\ddagger}$ score & Time & 14356.63 & 1.5 & 9060.23 & 47.13 & 0.000 & 0.51 \\
& Time*group & 20465.69 & 3.1 & 6457.77 & 33.59 & 0.000 & 0.59 \\
& Error & 13706.29 & 17.3 & 192.21 & - & - & - \\
\hline
\end{tabular}

ๆ, Hamilton anxiety scale; ¥, Hamilton depression scale; £, Difficulties in emotion regulation scale. 
three groups at pre-treatment post-treatment and followup phases. According to the results of this table, in the MCT group, the scores of anxiety, depression and difficulties in emotion regulation had significant differences at pre-treatment and post-treatment phases as well as at pre-treatment and follow-up phases. However, there was no significant difference in the scores between posttreatment and follow-up phases in this group. These results showed the effectiveness of MCT on the three variables as well as treatment-reliability over time. In the nortriptyline-treated group, there was a statistically significant difference in the scores of Hamilton anxiety and depression scales at the pre-test and post-test phases, while there was no significant difference at the post-test and follow-up phases, which implied the effect of nortriptyline on these variables over time. However, in the case of difficulties in emotion regulation scores, no significant difference was found at per- and post-treatment phases, indicating that nortriptyline had no effect on this variable $(\mathrm{P}<0.05)$. While, in the controls, there was no significant difference between scores in the three phases $(\mathrm{P}<0.05)$.

Dunnett's post-hoc test was performed to compare the MCT and nortriptyline-treated group with the control group. For the Hamilton anxiety scores, the mean difference of MCT and control group was (-16.27), and between the nortriptyline-treated and control group was $(-8.51)$; these differences were significant $(\mathrm{P}<0.05)$. Moreover, for the Hamilton depression scores, significant differences were found between the means of MCT and control group (-13.53), and between the nortriptylinetreated and control group (-10.25). However, no signifi- cant difference was found for the difficulties in emotion regulation scores between the groups $(\mathrm{P}<0.05)$.

Figure 1 shows linear trend analysis of changes in the scores of Hamilton anxiety and depression as well as difficulties in emotion regulation in the three groups at the phases of pre-treatment, post-treatment and three months after treatment. According to figure, there is a decreasing trend in the scores of all three variables of anxiety, depression and difficulties in emotion regulation in the metacognitive therapy group at the post-treatment and three months after treatment; this trend is higher compared to the nortriptyline treatment group. Moreover, there is a relatively decreasing trend in the scores of Hamilton anxiety and depression in the nortriptyline treatment group. However, this decreasing trend is not reliable at the follow-up three months after treatment. When it comes to the variable of difficulties in emotion regulation, there is a less decrease in the nortriptyline-treated group, but no reliability is observed at the follow-up session. Moreover, in the control group, there is no decreasing trend in the scores of the variables. Finally, Fig. 1 shows the higher short-term and long-term effects of the metacognitive therapy group compared to the other two groups in reducing the scores of all three variables.

The reliability of metacognitive therapy and nortriptyline treatment based on Cohen's effect size for the variables of Hamilton anxiety and depression as well as difficulties in emotion regulation showed that in metacognitive therapy the effect sizes of these three variables were $2.51,2.49$ and 2.80, respectively. However, in the nortriptyline treatment group, the effect sizes for Hamilton anxiety and depression variables and difficulties in emotional regulation were $0.21,0.14$ and 0.28 , respectively.

Table 4. Between-group pairwise comparisons of the scores of anxiety, depression and difficulties in emotion regulation.

\begin{tabular}{|c|c|c|c|c|c|c|c|c|c|c|}
\hline \multirow[b]{2}{*}{ Group } & \multirow[b]{2}{*}{ Group } & \multicolumn{3}{|c|}{ HAMA ${ }^{\pi}$ score } & \multicolumn{3}{|c|}{ HADS $^{\Psi}$ score } & \multicolumn{3}{|c|}{ DERS $^{\mathfrak{f}}$ score } \\
\hline & & $\mathbf{P}$ & SD & MD & $\mathbf{P}$ & SD & MD & $\mathbf{P}$ & SD & MD \\
\hline \multirow[t]{2}{*}{$\mathrm{MCT}^{\S}$} & Nortriptyline & -7.76 & 2.11 & 0.000 & 0.146 & 1.61 & -3.28 & -15.22 & 6.56 & 0.072 \\
\hline & Control & -16.26 & 2.26 & 0.000 & 0.000 & 1.64 & -13.53 & -11.26 & 6.67 & 0.295 \\
\hline Nortriptyline & Control & -8.50 & 2.18 & 0.001 & 0.000 & 1.65 & -10.25 & 4.05 & 6.77 & 1.000 \\
\hline
\end{tabular}

$\S$, Metacognitive therapy; $\uparrow$, Hamilton anxiety scale; $¥$, Hamilton depression scale; £, Difficulties in emotion regulation scale.

Table 5. pairwise comparisons of the scores of Hamilton anxiety and depression and difficulties in emotion regulation in three groups at pre-test post-test and follow-up phases.

\begin{tabular}{|c|c|c|c|c|c|c|c|}
\hline \multirow[b]{2}{*}{ Groups } & \multirow[b]{2}{*}{ Phases } & \multicolumn{2}{|c|}{ HAMA ${ }^{\pi}$ score } & \multicolumn{2}{|c|}{$\mathbf{A D S}^{ \pm}$score } & \multicolumn{2}{|c|}{ DERS $^{\mathfrak{t}}$ score } \\
\hline & & Posttest & Follow up & Posttest & Follow up & Posttest & Follow up \\
\hline \multirow[t]{2}{*}{$\mathrm{MCT}^{\S}$} & Pretest & $18.00^{*}$ & $18.82 *$ & $12.23 *$ & $12.88^{*}$ & $52.64 *$ & $59.05^{*}$ \\
\hline & Posttest & - & 0.82 & - & 0.64 & - & 6.41 \\
\hline \multirow[t]{2}{*}{ Nortriptyline } & Pretest & $11.06^{*}$ & $10.18^{*}$ & $5.56^{*}$ & $4.18^{*}$ & 9.50 & 6.93 \\
\hline & Posttest & - & -0.87 & - & -1.3 & - & -2.56 \\
\hline \multirow[t]{2}{*}{ Control } & Pretest & -0.53 & -1.000 & -1.06 & -0.60 & -1.53 & 0.26 \\
\hline & Posttest & - & -0.46 & - & 0.46 & - & 1.80 \\
\hline
\end{tabular}

*, $\mathrm{P}<0.05 ; \S$, Metacognitive therapy; $\uparrow$, Hamilton anxiety scale; $¥$, Hamilton depression scale; $£$, Difficulties in emotion regulation scale. 


\section{Discussion}

The present study was intended to perform a controlled comparative investigation of metacognitive psychotherapy and nortriptyline treatment for improving anxiety, depression and difficulties in emotion regulation in patients with FD in short-term and three months after treatment (longterm). According to the results, MCT showed a statistically significant improvement in the symptoms of anxiety, depression, and difficulties in emotion regulation in patients with FD at post-treatment and three months after treatment. The MCT was better in improving anxiety symptoms than nortriptyline treatment and compared to the control group. There was no statistically significant difference in improving depression symptoms between the MCT and nortriptyline treatment groups. However, the MCT group showed a significant difference in improving depression symptoms compared to the control group. In addition, no significant difference was found for improving the difficulties in emotion regulation among the groups. Moreover, the effect size of MCT on improving the anxiety, depression, and difficulties in emotion regulation was larger than nortriptyline treatment.

To the best of our knowledge, there has been no clinical trial of the effects of MCT on the improvement of symptoms of depression, anxiety and difficulties in emotional regulation in patients with FD and other functional gastrointestinal disorders.

Anxiety symptoms and anxiety disorders have a high prevalence in patients with FD (Tanum, \& Malt, 2001; Mahadeva, \& Goh, 2011; Filipović et al., 2013), and a higher level of anxiety increases the dyspeptic discomfort in these patients ( $\mathrm{Li}$ et al., 2002; Van Oudenhove et al., 2007; Aro et al., 2009; Wu, 2012). Furthermore, the results of previous studies have shown the positive impact of MCT on generalized anxiety disorder and anxiety symptoms (Wells, 1999; Wells, \& King, 2006; Wells, 2007; Wells et al., 2010; Wells, 2011).
Anxiety treatment protocol of metacognitive psychotherapy focuses on the elimination of cognitive-attentional syndrome (CAS). This syndrome as a common thought pattern in people with mental disorders has a repetitive and ruminative nature. Moreover, CAS causes excessive conceptual processing of worry and attentional bias towards risk and threat-related stimuli in patients with Generalized Anxiety Disorders (GAD).

In the metacognitive model, it seems that the worry in FD patients with comorbidity of anxiety as the GAD patients is associated with negative and positive metacognitive thoughts and beliefs. Additionally, MCT focuses on modifying and removing positive and negative metacognitive beliefs. Positive metacognitive beliefs about worry are associated with the usefulness and effectiveness of worry and other metacognitive strategies. Negative metacognitive beliefs express the uncontrollability and danger of worry that exacerbate the anxiety-related physiological, cognitive, emotional and behavioral symptoms. Finally, both the positive and negative metacognitive beliefs perpetuate and exacerbate CAS.

Accordingly, MCT based on modifying CAS, changing metacognitive beliefs and developing alternative strategies for experiencing or coping with internal events can remove the symptoms of worry, anxiety, threat monitoring and self-focused attention, and perpetuating factors of symptoms in a long-term period. Moreover, compared to the medication or control group, MCT can have a reliable effect on reducing anxiety symptoms of FD patients.

The treatment protocol used in this study has not focused on depressive symptoms, as well as the rumination and metacognitive beliefs corresponding to depressive disorder, so there was limited efficacy on these symptoms; moreover, no statistically significant difference was found compared to the case of taking nortriptyline as a tricyclic antidepressant. Since the treatment protocol was based on cognition, metacognition and metacognitive strategies, its effects on emotion regulation and management had some

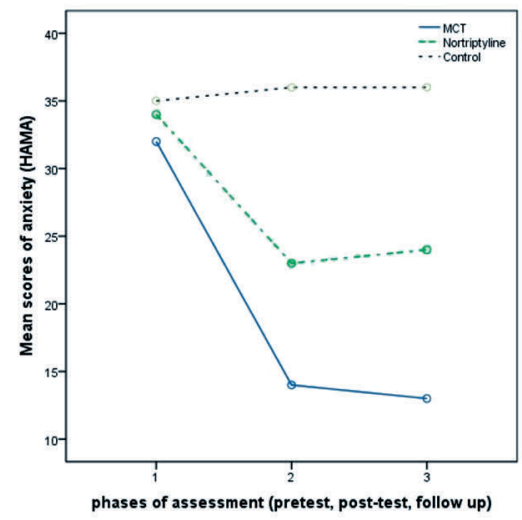

(a)

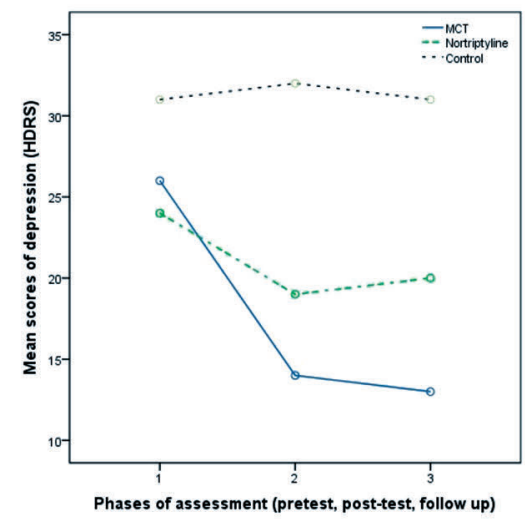

(b)

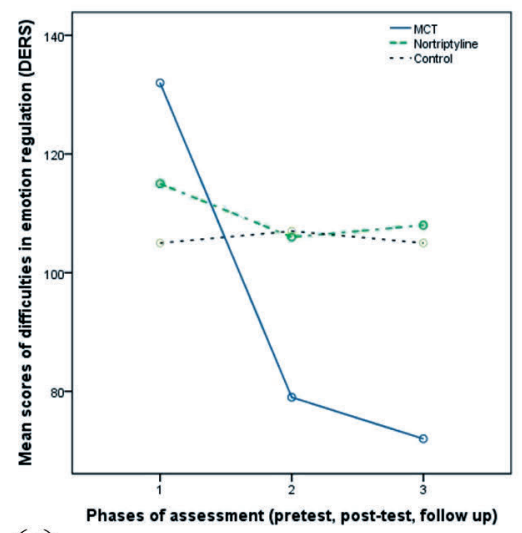

(c)

Figure 1. Linear trend analysis of the scores of (a) HAMA, (b) HDRS and (c) DERS for three groups in pretest, post-test, and follow-up. 
limitations. However, some techniques used in this treatment, such as detached mindfulness, can improve emotion dysregulation (Guendelman, Medeiros, \& Rampes, 2017).

In the general sense, detached mindfulness means accepting thoughts and feelings without the need to avoid or judge them; this increases the capacity for acceptance of thought, feelings and emotional experience (Wells, 2002; Wells, 2011). Because of using only a few emotionfocused techniques in this study, these techniques had only a limited effect on the patients' emotion regulation. Therefore, only the within-group effects of the MCT were statistically significant in the short-term and three months after treatment.

The findings of this study are in agreement with the findings of previous researches that investigate the effectiveness of other psychotherapies such as flexible coping psychotherapy in reducing dyspeptic/anxiety symptoms (Cheng et al., 2007) and intensified medical treatment with CBT in reducing depression/anxiety symptoms (Haag et al, 2007).

On the one hand, the similarity between MCT and cognitive-behavioral therapies has led to the results that are similar to the results of previous researches in reducing the psychological symptoms of FD patients. Indeed, the similarity between MCT and cognitive-behavioral therapies correspond to the structural-based utilization of cognitive techniques for challenging metacognitive beliefs and behavioral techniques such as behavioral experiment for eliminating behavioral avoidance.

On the other hand, in comparison with other psychotherapy strategies, such as cognitive-behavioral therapy and other types of analytical psychotherapy based on resolving interpersonal conflicts, the MCT model reduces the physical symptoms of anxiety by training distraction from stressful stimuli and worry postponement for treating FD symptoms. That is, MCT aims at mindfulness training to actively engage the patient in therapeutic process. Moreover, MCT helps avoiding negative thoughts without engaging the patient in his/her thoughts and their evaluation.

Metacognitive therapy causes an increase in mindfulness in FD patients. This is realized by observing and paying attention to internal experiences (such as thoughts and emotions), with acceptance and a non-judgmental attitude. The patients understand their inner and outer realities truthfully without any distortion. Their ability to expose a wide range of experiences, emotions and unpleasant thoughts is increased. Moreover, detached mindfulness prevents ineffective coping behaviors and automatic responses of the sympathetic system to stressful experiences. This is done by increasing self-monitoring of physical activity, as well as awareness to emotion and body mechanisms and behavioral self-regulation.

Unlike other cognitive therapies, instead of challenging intrusive thoughts and dysfunctional beliefs, MCT changes the way of dealing with thoughts to prevent re- sistance or complex perceptual analysis. Consequently, maladaptive thinking strategies about anxiety and inflexible threat-monitoring are eliminated. Therefore, the recognition of negative beliefs and the way of reacting to these beliefs, which are considered in other cognitive models, cannot explain the thinking patterns and the strategies for responding to thoughts in MCT model. In addition, this model of therapy considers the factors that control thoughts and change the state of mind processing. Patients with FD can gradually reduce the need for controlling thoughts and break the anxiety processing link. Moreover, patients with FD become more aware of the inefficiency of using strategies such as thought suppression to control thoughts and seek alternative coping techniques to improve anxiety-related beliefs. Finally, this model increases the patients' awareness of the processes in metacognitive processing system and processing thoughts in a metacognitive manner.

As the results showed, even after three months followup, MCT has a positive effect on reducing anxiety/depression and improvement in the emotional regulation of FD patients. This results from changing the mental processing system and using cognitive-behavioral techniques to challenge and eliminate positive and negative metacognitive beliefs. In fact, MCT improve the psychological symptoms of FD patients in short sessions, so, MCT strategies as more comprehensive therapy versus other cognitive psychotherapy is appropriate for outpatient treatment centers as well as gastrointestinal clinics when it comes to using cognitive-behavioral and experimental techniques. The present study indicates the short- and long-term effectiveness of MCT in reducing psychological symptoms, especially anxiety, in patients with FD compared to the conventional drug therapy. Therefore, MCT can be applied as a treatment option for improving the psychological symptoms of FD patients in clinical settings.

The ratio of male patients visited the clinic during the study was lower than female patients; this limits the generalizability of the findings to the males. Moreover, most of the participants had low social-economic and educational status, so this limitation should be considered in future studies. In this treatment protocol, the use of emotion-focused techniques was also limited, and because of the limited sample size and high possibility of long-term dropouts, only short-term follow-up sessions were considered.

It is suggested that larger samples of homogeneous genders and various socioeconomic and educational statuses can extend the findings of the present study in future researches. Furthermore, the effects of emotion-focused psychotherapies can be studied on improving the symptoms of FD patients in longer-term follow-up phases.

\section{Conclusions}

The results of the current study showed that a combination of metacognitive psychotherapy and conventional 
medical treatment could improve the symptoms of anxiety, depression and difficulties in emotion regulation of FD patients in short- and long-term follow up. Moreover, MCT had better effectiveness on FD patients compared to the medication and controls in improving anxiety symptoms.

\section{References}

American Psychiatric Association. (2013). Diagnostic and statistical manual of mental disorders ( $5^{\text {th }}$ ed.). DSM-5®. American Psychiatric Pub.

Aro, P., Talley, N. J., Ronkainen, J., Storskrubb, T., Vieth, M., Johansson, S. E., Boiling-sternevald, L., \& Agréus, L. (2009). Anxiety is associated with uninvestigated and functional dyspepsia (Rome III criteria) in a Swedish population-based study. Gastroenterology, 137(1), 94-100.

Bagherian, R., Pourkazem, T., Nouri, A., \& Adibi, P. (2009). The effects of stress management training on symptoms of medical treatment-resistant functional dyspepsia. Govaresh, 14(1), 15-22.

Batebi, S., Masjedi Arani, A., Jafari, M., Sadeghi, A., Saberi Isfeedvajani, M., davazdahemami, M.H. (2019). Validity and Reliability of the Persian Version of Leeds Dyspepsia Questionnaire. Galen Medical Journal, 8, e1609. doi:10.31661/ gmj.v8i0.1609

Calvert, E. L., Houghton, L. A., Cooper, P., Morris, J., \& Whorwell, P. J. (2002). Long-term improvement in functional dyspepsia using hypnotherapy. Gastroenterology, 123(6), 1778-1785.

Chen, T. S., Luo, J. C., \& Chang, F. Y. (2010). Psychosocialspiritual factors in patients with functional dyspepsia: a comparative study with normal individuals having the same endoscopic features. European Journal of Gastroenterology \& Hepatology, 22(1), 75-80.

Cheng, C., Yang, F. C., Jun, S., \& Hutton, J. M. (2007). Flexible coping psychotherapy for functional dyspeptic patients: a randomized, controlled trial. Psychosomatic Medicine, 69(1), 81-88

Cusin, C., Yang, H., Yeung, A., \& Fava, M. (2009). Rating scales for depression. In: Handbook of clinical rating scales and assessment in psychiatry and mental health. Humana Press, Totowa, NJ. Springer. 7-35.

Davis, R. N., \& Valentiner, D. P. (2000). Does meta-cognitive theory enhance our understanding of pathological worry and anxiety? Personality and Individual Differences, 29(3), 513-526.

Dehghanizade, Z., Zargar, Y., Honarmand, M. M., Kadkhodaie, A., \& Baygi, M. E. (2015). The effectiveness of cognitive behavior stress management on functional dyspepsia symptoms. Journal of Advances in Medical Education \& Professionalism, 3(2), 45.

Drossman, D. A., Toner, B. B., Whitehead, W. E., Diamant, N. E., Dalton, C. B., ..., Bangdiwala, S.I. (2003). Cognitivebehavioral therapy versus education and desipramine versus placebo for moderate to severe functional bowel disorders. Gastroenterology, 125(1), 19-31.

Ebrahimi, A., Kheyr, Z., Nasiri, H., \& Barnamanesh, A. (2017). Reliability, validity, factor and discrimination power analysis of young mania rating scale among unipolar, bipolar depression and healthy people. Jundishapur Scientific Medical Journal, 16(3), 333-344.
Faramarzi, M., Azadfallah, P., Book, H. E., Tabatabaei, K. R., Taheri, H., \& Shokri-shirvani, J. (2013). A randomized controlled trial of brief psychoanalytic psychotherapy in patients with functional dyspepsia. Asian Journal of psychiatry, 6(3), 228-234.

Filipović, B. F., Randjelovic, T., Ille, T., Markovic, O., Milovanović, B., Kovacevic, N., \& Filipović, B. R. (2013). Anxiety, personality traits and quality of life in functional dyspepsia $\square$ suffering patients. European Journal of Internal Medicine, 24(1), 83-86.

First, M. B. (2014). Structured clinical interview for the DSM (SCID). The encyclopedia of clinical psychology, 1-6.

Gratz, K. L., \& Roemer, L. (2004). Multidimensional assessment of emotion regulation and dysregulation: Development, factor structure, and initial validation of the difficulties in emotion regulation scale. Journal of Psychopathology and Behavioral Assessment, 26(1), 41-54.

Gross, J. J. (2001). Emotion regulation in adulthood: Timing is everything. Current directions in psychological science, 10(6), 214-219.

Gross, J. J., \& Thompson, R. A. (2007). Emotion regulation: Conceptual foundations Handbook of emotion regulation. The Guilford Press, New York, NY, US.

Guendelman, S., Medeiros, S., \& Rampes, H. (2017). Mindfulness and emotion regulation: Insights from neurobiological, psychological, and clinical studies. Frontiers in Psychology, 8, 220-243. doi: 10.3389/fpsyg.2017.00220.

Haug, T. T., Wilhelmsen, I., Svebak, S., Berstad, A., \& Ursin, H. (1994). Psychotherapy in functional dyspepsia. Journal of Psychosomatic Research, 38(7), 735-744.

Haug, T. T. (2002). Cognitive therapy in functional dyspepsia. International Congress Series, 1241, 127-130.

Haag, S., Senf, W., Tagay, S., Langkafel, M., Braun $\square$ Lang, U., Pietsch, A., ..., \& Holtmann, G. (2007). Is there a benefit from intensified medical and psychological interventions in patients with functional dyspepsia not responding to conventional therapy? Alimentary Pharmacology \& Therapeutics, 25(8), 973-986.

Hamilton, M. A. X. (1959). The assessment of anxiety states by rating. British Journal of Medical Psychology 32(1), 50-55.

Hamilton M. (1960). A rating scale for depression. Journal of Neurology, Neurosurgery \& Psychiatry, 23(1), 56-62.

Hamilton, J., Guthrie, E., Creed, F., Thompson, D., Tomenson, B., Bennett, R., ..., \& Liston, R. (2000). A randomized controlled trial of psychotherapy in patients with chronic functional dyspepsia. Gastroenterology, 119(3), 661-669.

Jones, M. P., Dilley, J. B., Drossman, D., \& Crowell, M. D. (2006). Brain-gut connections in functional GI disorders: anatomic and physiologic relationships. Neurogastroenterology \& Motility, 18(2), 91-103.

Li, Y., Nie, Y., Sha, W., \& Su, H. (2002). The link between psychosocial factors and functional dyspepsia: an epidemiological study. Chinese medical journal, 115(7), 1082-1084.

Mahadeva, S., \& Goh, K. L. (2011). Anxiety, depression and quality of life differences between functional and organic dyspepsia. Journal of Gastroenterology and Hepatology, 26, 49-52.

Mahadeva, S., Chan, W. K., Mohazmi, M., Sujarita, R., \& Goh, K. L. (2011). Validation study of the Leeds Dyspepsia Questionnaire in a multi $\square$ ethnic Asian population. Journal of Gastroenterology and Hepatology, 26(11), 1669-1676.

Maier, W., Buller, R., Philipp, M., \& Heuser, I. (1988). The Hamilton Anxiety Scale: reliability, validity and sensitivity 
to change in anxiety and depressive disorders. Journal of Affective Disorders, 14(1), 61-68.

Mennin, D. S., Heimberg, R. G., Turk, C. L., \& Fresco, D. M. (2002). Applying an emotion regulation framework to integrative approaches to generalized anxiety disorder. Clinical Psychology: Science and Practice, 9(1), 85-90.

Mennin, D. S., Turk, C. L., \& Heimberg, R. G. (2004). Clinical presentation and descriptive psychopathology of generalized anxiety disorder. In: Generalized anxiety disorder: advances in research and practice. New York: Guilford Press. p, 3-28.

Mennin, D. S., Heimberg, R. G., Turk, C. L., \& Fresco, D. M. (2005). Preliminary evidence for an emotion dysregulation model of generalized anxiety disorder. Behaviour Research and Therapy, 43(10), 1281-1310.

Mennin, D. S., Holaway, R. M., Fresco, D. M., Moore, M. T., \& Heimberg, R. G. (2007). Delineating components of emotion and its dysregulation in anxiety and mood psychopathology. Behavior Therapy, 38(3), 284-302.

Moayyedi, P., Duffett, S., Braunholtz, D., Mason, S., Richards, I. D., Dowell, A. C., \& Axon, A. T. (1998). The Leeds Dyspepsia Questionnaire: a valid tool for measuring the presence and severity of dyspepsia. Alimentary Pharmacology \& Therapeutics, 12(12), 1257-1262.

Mokhber, N., AzarPajooh, M. R., \& Asghari, P. N. (2013). Effect of citalopram on depression and cognitive functions of stroke patients. Journal of Sabzevar University of Medical Sciences, 20(1): 6-14.

Molazadeh, J., \& Yavari, A. H. (2016). Study of the mediating role of emotion regulation difficulties in the relationship between exposure to traumatic events and risky sexual behavior among substance abusers. Yafte, 18(3):78-87.

Orive, M., Barrio, I., Orive, V. M., Matellanes, B., Padierna, J. A., Cabriada, J., ... \& Quintana, J. M. (2015). A randomized controlled trial of a 10-week group psychotherapeutic treatment added to standard medical treatment in patients with functional dyspepsia. Journal of Psychosomatic Research, 78(6), 563-568.

Sahan, H. E., Yildirim, E. A., Soylu, A., Tabakci, A. S., Cakmak, S., \& Erkoc, S. N. (2018). Comparison of functional dyspepsia with organic dyspepsia in terms of attachment patterns. Comprehensive Psychiatry, 83, 12-18.

Salmani, B., Hasani, J., Mohammad-Khani, S., \& Karami, G. R. (2014). The efficacy of metacognitive therapy on metacognitive beliefs, metaworry and the signs and symptoms of patients with generalized anxiety disorder. KAUMS Journal (FEYZ), 18(5), 428-439.

Salters-Pedneault, K., Tull, M. T., \& Roemer, L. (2004). The role of avoidance of emotional material in the anxiety disorders. Applied and Preventive Psychology, 11(2), 95-114.

Salters-Pedneault, K., Roemer, L., Tull, M. T., Rucker, L., \& Mennin, D. S. (2006). Evidence of broad deficits in emotion regulation associated with chronic worry and generalized anxiety disorder. Cognitive Therapy and Research, 30(4), 469-480.

Schmulson, M. J., \& Drossman, D. A. (2017). What is new in Rome IV. Journal of Neurogastroenterology and Motility, 23(2), 151.

Taché, Y., \& Bonaz, B. (2007). Corticotropin-releasing factor receptors and stress-related alterations of gut motor function. The Journal of Clinical Investigation, 117(1), 33-40.

Talley, N. J., Ruff, K., Jiang, X., \& Jung, H. K. (2008). The Rome III classification of dyspepsia: will it help research? Digestive Diseases, 26(3), 203-209.

Tanum, L., \& Malt, U. F. (2001). Personality and physical symptoms in nonpsychiatric patients with functional gastrointestinal disorder. Journal of Psychosomatic Research, 50(3), 139-146.

Todorova, K. S., \& Velikova, V. S. (2012). The validity of the Hamilton depression rating scale as a screening and diagnostic instrument for depression in patients with epilepsy. Journal of IMAB-Annual Proceeding Scientific Papers, 18(3), 305-307.

Turk, C. L., Heimberg, R. G., \& Luterek, J. A. (2005). Delineating emotion regulation deficits in generalized anxiety disorder: A comparison with social anxiety disorder. Cognitive Therapy and Research, 29(1), 89-106.

Van Oudenhove, L., Vandenberghe, J., Geeraerts, B., Vos, R., Persoons, P., Demyttenaere, K., Fischler, B., \& Tack, J. (2007). Relationship between anxiety and gastric sensorimotor function in functional dyspepsia. Psychosomatic Medicine, 69(5), 455-463.

Wells, A. (1999). A metacognitive model and therapy for generalized anxiety disorder. Clinical Psychology \& Psychotherapy: An International Journal of Theory \& Practice, 6(2), $86-95$.

Wells, A. (2002). Emotional Disorders and Metacognition.: Innovative Cognitive Therapy. John Wiley \& Sons. New York, NY, US.

Wells, A., \& King, P. (2006). Metacognitive therapy for generalized anxiety disorder: An open trial. Journal of Behavior Therapy and Experimental Psychiatry, 37(3), 206-212.

Wells, A. (2007). Cognition about cognition: Metacognitive therapy and change in generalized anxiety disorder and social phobia. Cognitive and Behavioral Practice, 14(1), 18-25.

Wells, A., Welford, M., King, P., Papageorgiou, C., Wisely, J., $\&$ Mendel, E. (2010). A pilot randomized trial of metacognitive therapy vs applied relaxation in the treatment of adults with generalized anxiety disorder. Behaviour Research and Therapy, 48(5), 429-434.

Wells, A. (2011). Metacognitive therapy for anxiety and depression. Guilford press. New York, NY, US.

Wu, J. C. (2012). Psychological co-morbidity in functional gastrointestinal disorders: epidemiology, mechanisms and management. Journal of Neurogastroenterology and Motility, 18(1), 13-18. 